\title{
Repeptization by Dissolution in a Colloidal System of Iron(III) Pyrophosphate
}

\author{
Y. Mikal van Leeuwen, ${ }^{* \dagger}{ }^{\dagger}$ Krassimir P. Velikov, ${ }^{\dagger}$ and Willem K. Kegel ${ }^{* \dagger}$ \\ †Van 't Hoff Laboratory for Physical and Colloidal Chemistry, Utrecht University, Padualaan 8, 3584 CH Utrecht, The Netherlands \\ ${ }^{\ddagger}$ Unilever Research and Development Vlaardingen, Olivier van Noortlaan 120, 3133 AT Vlaardingen, The Netherlands
}

Supporting Information

ABSTRACT: Repeptization (redispersion) from an aggregated state is usually only possible in charge-stabilized colloidal systems if the system is either coagulated in the secondary minimum of the interaction potential or if the system cannot settle completely into the primary minimum. In this work, we analyze the zeta potential, conductivity, and long-term stability of colloidal systems of iron(III) pyrophosphate and surprisingly find that the system seems to defy conventional wisdom as it can be repeptized from its coagulated state regardless of aging time and background ions. Moreover, after having been stored for up to a month in $2 \mathrm{M} \mathrm{NaCl}$, dialysis of iron pyrophosphate will yield a colloidal dispersion that is actually

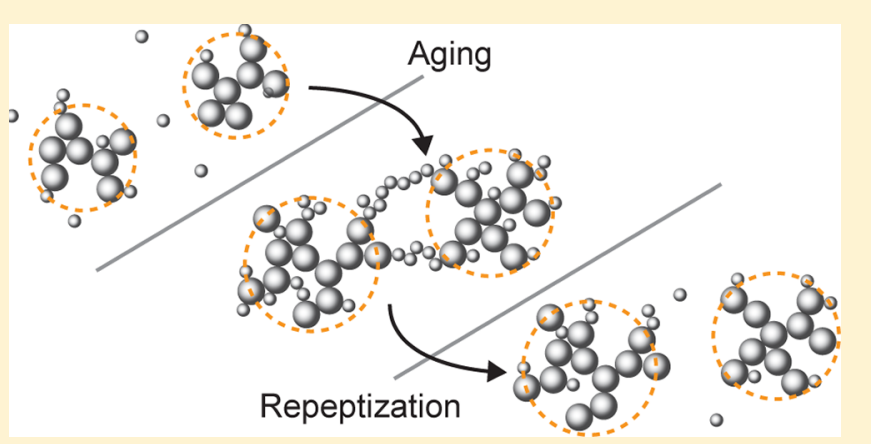
stable for a longer period of time than a fresh system with background electrolyte removed.

\section{INTRODUCTION}

Destabilization and coagulation in the primary minimum of the interaction potential occurs when colliding particles overcome the repulsion and cross the stabilizing barrier; see Figure 1. Once trapped, lowering the ionic strength will only repeptize (redisperse) the particles if there is a distance of closest approach (or cutoff distance) between the particles, otherwise repeptization from the deep primary minimum will be

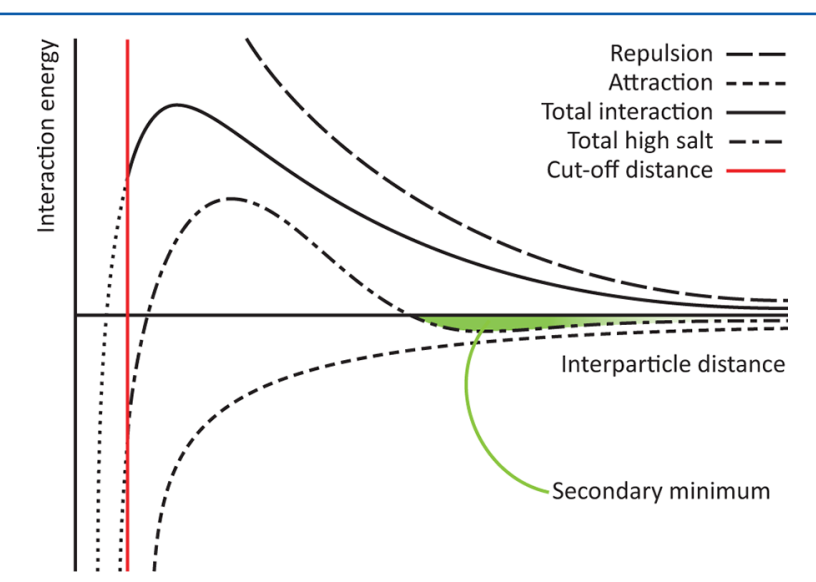

Figure 1. Schematic representations of interparticle interaction potentials in an electrocratic system: the double layer repulsion (long dash) and the van der Waals attraction (short dash) together yield the total interaction potential either without (solid line) or with (dash-dotted line) a secondary minimum depending on the repulsion strength. With the introduction of a cutoff distance, the deepest part of the primary minimum is not available to the particles (dotted lines). impossible. ${ }^{1}$ A possible cause for such a cutoff distance in an electrocratic (charge-stabilized) system arises if interparticle interactions during a Brownian collision occur under constant surface charge instead of constant surface potential, as the reequilibration needed for a constant potential takes place in a timespan orders of magnitude larger than that of the collisions. This implies that the cutoff distance will vanish over time with equilibration, explaining why aged systems cannot be repeptized as is indeed often observed. ${ }^{2,3}$ Other possible causes for a cutoff distance include surface roughness and the presence of the hydration layer itself, but these will also vanish over time by re-equilibration or sintering. ${ }^{1,4-6}$ Note that we are working with a system that contains no stabilizing agents such as surfactants or polymers, making steric stabilization (another common cause for a distance of closest approach) highly unlikely.

While repeptization is important from a practical viewpoint as it governs the ability for colloidal dispersions to be dried, stored, and redispersed, it is almost exclusively studied when going from the dried to the dispersed state, ${ }^{4,7}$ comprising only a minor part of the phenomenon. However, current literature agrees that while repeptization from the primary minimum can be experimentally realized when coagulation is induced by monovalent background ions and short aging times, the repeptization of systems aggregated by polyvalent ions is not possible by simply lowering the ionic strength of the medium: it needs additional means such as addition of surfactants ${ }^{8}$ or

Received: September 12, 2012

Revised: November 13, 2012

Published: November 14, 2012 
complexing agents. ${ }^{9}$ In this work, we further investigate the long-term stability of colloidal systems of iron pyrophosphate and unexpectedly find that they can be repeptized independent of aging time and valence of background ions and without any further additives.

Iron(III) pyrophosphate (ferric pyrophosphate, $\mathrm{FePP}_{\mathrm{i}}$ ) is the only iron-containing compound without color, explaining why it is commercially available as a food additive and mineral supplement. It is an easily concealable material that is useful for fighting iron deficiency due to its good bioaccessibility. ${ }^{10-12}$

We have shown before that, when preparing colloidal iron pyrophosphate, the material precipitates into nanometer sized particles that immediately aggregate into $\sim 200 \mathrm{~nm}$ clusters. ${ }^{13}$ While these clusters remain in dispersion for days, they slowly grow until the system is macroscopically aggregated. Our calculations showed that that the initial instability (resulting in the aggregation into small clusters) is caused by the higher ionic strength at the start of the precipitation. When precipitation is complete, the ionic strength is lower, and the clusters have become (meta)stable. We also observed that the system consists of two particle sizes: $20 \mathrm{~nm}$ particles in the clusters and $5 \mathrm{~nm}$ particles both in the clusters and separate in solution; see Figure 2. These results, together with cryo transmission

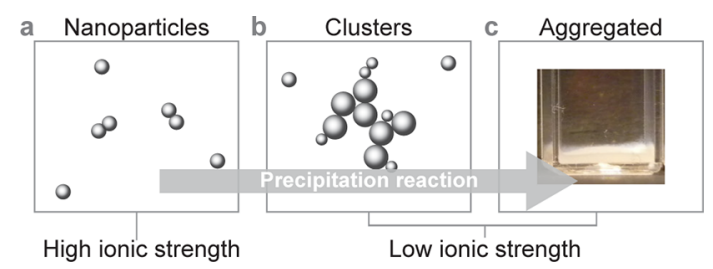

Figure 2. Three steps in the preparation and aging of the system: $\mathrm{FePP}_{\mathrm{i}}$ forms nanoparticles upon precipitation (a) that immediately form into finite-sized clusters (b). Eventually, these clusters will aggregate macroscopically (c). As $\mathrm{FePP}_{\mathrm{i}}$ is prepared from ionic precursor materials, the ionic strength at the start of the precipitation (a) is higher than when precipitation is complete $(b, c)$.

electron microscopy (TEM) analysis, lead us to propose that the individual $5 \mathrm{~nm}$ particles caused the further aggregation by linking the clusters together.

In the current work, we further investigate the colloidal stability of $\mathrm{FePP}_{\mathrm{i}}$ and find that (1) we can prepare a system that remains stable for more than a year and that (2) we are able to repeptize a system that has been coagulated under circumstances that should not allow for repeptization as discussed above.

\section{EXPERIMENTAL SECTION}

Preparation. Samples were prepared as reported elsewhere. ${ }^{13}$ In brief, $0.857 \mathrm{mmol}$ of $\mathrm{FeCl}_{3} \cdot 6 \mathrm{H}_{2} \mathrm{O}$ (Sigma Aldrich) in $50 \mathrm{~mL}$ of water was added dropwise in about $15 \mathrm{~min}$ to $0.643 \mathrm{mmol}$ of $\mathrm{Na}_{4} \mathrm{P}_{2} \mathrm{O}_{7} \cdot 10 \mathrm{H}_{2} \mathrm{O}$ (Acros) in $100 \mathrm{~mL}$ water while stirring. For the dialysis experiments, dispersions were dialyzed using Spectra/Por 2 Dialysis Membrane, molecular weight cutoff (MWCO) 12-14.000 Da, corresponding to roughly $1.5 \mathrm{~nm}$. In each dialysis run, $20 \mathrm{~mL}$ of dispersion in a dialysis tube was stored in $500 \mathrm{~mL}$ of medium, which was replaced with new water every day. For the repeptization experiment, $\mathrm{NaCl}, \mathrm{LiCl} \cdot x \mathrm{H}_{2} \mathrm{O}, \mathrm{KCl}, \mathrm{MgCl}_{2} \cdot 6 \mathrm{H}_{2} \mathrm{O}$, and $\mathrm{AlCl}_{3} \cdot 6 \mathrm{H}_{2} \mathrm{O}$ were all obtained from Sigma Aldrich. All chemicals were used as received, solutions were prepared in water deionized using a Millipore Synergy water purification system, which was also used for the dialysis medium.
Analysis. Dynamic light scattering (DLS) and electrophoretic mobility analysis measurements were performed using a Malvern Instruments Zetasizer Nano series machine operating in backscatter mode at $25{ }^{\circ} \mathrm{C}$ with $5 \mathrm{~min}$ of equilibration time. Samples for the DLS time series were filtered over a Minisart disposable cellulose acetate filter $(0.45 \mu \mathrm{m}$ poresize, $16555-\mathrm{K})$ and stored in a plastic disposable DLS cuvette sealed with parafilm. Samples for electrophoretic mobility measurements were taken from the stock sample, DLS analysis of these samples showed no difference with the sealed DLS samples. For cryo-TEM, one drop of unaltered dispersion was placed on a cryoTEM grid and blotted for $1 \mathrm{~s}$ before being quenched in liquid ethane. Samples were stored in liquid nitrogen before being analyzed using a Tecnai 20 electron microscope.

\section{RESULTS}

A freshly prepared dispersion of iron pyrophosphate was divided into two portions. One solution was left unchanged (containing $17 \mathrm{mM} \mathrm{NaCl}$ and labeled low salt or LS), and to the other, $\mathrm{NaCl}, \mathrm{KCl}$, or $\mathrm{LiCl}$ was added to obtain a $2 \mathrm{M}$ solution (labeled high salt or HS). Samples were taken from both dispersions at three intervals: (i) one hour, (ii) 19 days, and (iii) 35 days after preparation; see Figure 3 for a schematic overview of the sample preparation. These samples were dialyzed for three or six days and followed over time by means of DLS analysis.

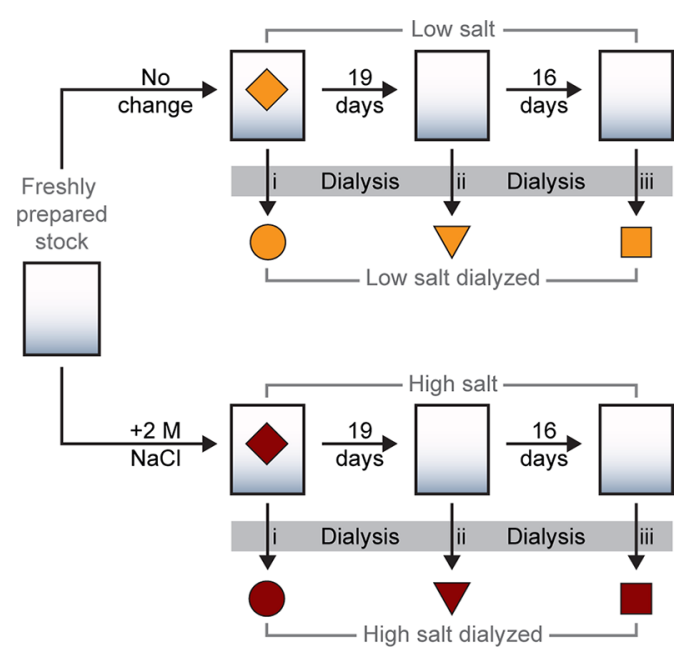

Figure 3. Preparation scheme. The freshly prepared dispersion of iron pyrophosphate (left) is divided into two portions: one is left unchanged (top row, low salt); one is adjusted to $2 \mathrm{M}$ (bottom row, high salt). Both are aged and dialyzed at three intervals. The addition of $2 \mathrm{M}$ salt causes immediate sedimentation. Symbols correspond to those in Figure 4.

For clarity, we divide our results into the description of two phenomena in this section: (1) the repeptization of aggregated systems and (2) the long-term stability of (dialyzed) systems.

Repeptization of Aggregated Systems. As can be seen in Figure 4a, all dialyzed samples (either low salt or $\mathrm{NaCl}$ high salt) were repeptized independent of waiting time or salt concentration. The low salt samples have cluster sizes of around $200 \mathrm{~nm}$ after dialysis, while the high salt samples show sizes of around $500 \mathrm{~nm}$ immediately after dialysis, which slowly decrease to $400 \mathrm{~nm}$ in days. Note that these sizes should not be interpreted as absolute numbers; polydispersity is very high in these samples $(>30 \%)$. The unchanged initial low salt system shows the characteristic growth found in previous experiments ${ }^{13}$ and is completely aggregated after 20 days. The cryo- 

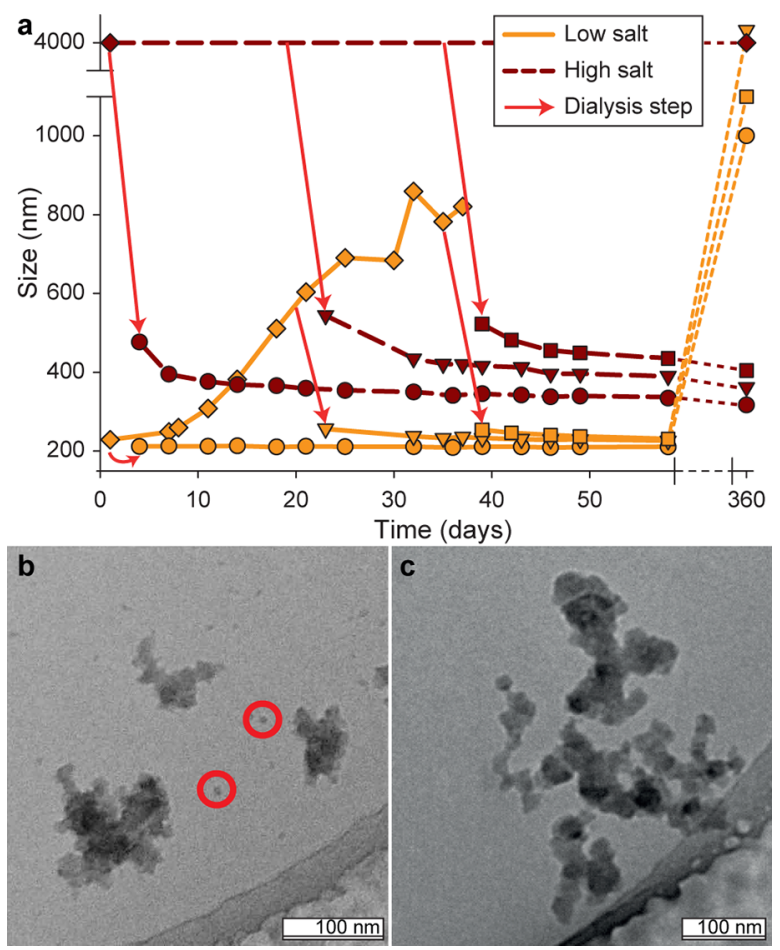

Figure 4. Growth of the cluster size over time for the $\mathrm{NaCl}$ repeptization experiment measured by dynamic light scattering (a). The dispersion without any added salt shows similar growth as before (light $\diamond$ ). The dispersion with an additional $2 \mathrm{M} \mathrm{NaCl}$ could not be accurately analyzed due to the macroscopic size of the particles but is indicated for reference (dark $\diamond)$. Dialysis (three days) performed immediately $(O), 19$ days $(\nabla)$, and 35 days $(\square)$ after preparation, red arrows indicate dialysis steps. Symbols correspond to those in Figure 3. Cryo-TEM analysis of the low salt system dialyzed for three days immediately after dialysis (b) shows no difference with previous images, two individual $5 \mathrm{~nm}$ are indicated with circles. After six days of dialysis, the $5 \mathrm{~nm}$ particles are absent (c).

TEM micrograph depicted in Figure $4 \mathrm{~b}$ shows no change in size or number of nanoparticles after 3 days of dialysis, but after 6 days of dialysis, the unbound, approximately $5 \mathrm{~nm}$ sized particles are no longer present (Figure 4c). The pore size of the dialysis membrane is sufficiently small to prevent the $5 \mathrm{~nm}$ particles from leaking out. Longer dialysis times and different background cations $\left(\mathrm{K}^{+}, \mathrm{Li}^{+}\right)$yield similar results (not shown).

Table 1 shows that high salt systems with $\mathrm{Mg}^{2+}$ and $\mathrm{Fe}^{3+}$ as background ions can also be repeptized although they result in larger cluster sizes. It should be noted here that the $\mathrm{Fe}^{3+}$ system is not aged with a $1 \mathrm{M}$ excess, as adding such an amount of $\mathrm{Al}^{3+}$ or $\mathrm{Fe}^{3+}$ completely dissolves the particles. This is most likely due to a change in $\mathrm{pH}$; see Supporting Information for details. Because an excess of $0.2 \mathrm{mM}$ arguably leads to aggregation in the secondary minimum instead of the primary, the system was aged for one month before dialysis to allow for further aggregation into the primary minimum.

Dialysis Time and Long-Term Stability. While all systems were initially repeptized, most systems had aggregated completely roughly one year after preparation. Figure 4a shows that the only samples that were still stable after one year were those prepared by a 3 day dialysis of the high salt system, independent of waiting time. Note that the high salt systems that were dialyzed for 6 days have destabilized as well after one year. The results are summarized in Table 2, the only preparation route that leads to systems with long-term (more
Table 1. Dynamic Light Scattering, Zeta Potential, and Conductivity Results of the High Salt Systems with Multivalent Ions

\begin{tabular}{cllcc}
\multicolumn{1}{c}{ sample } & $\begin{array}{c}\text { dialysis } \\
\text { duration }\end{array}$ & $\begin{array}{c}\text { size } \\
(\mathrm{nm})\end{array}$ & $\begin{array}{c}\text { zeta potential } \\
(\mathrm{mV})\end{array}$ & $\begin{array}{c}\text { conductivity } \\
(\mathrm{mS} / \mathrm{cm})\end{array}$ \\
\hline $1 \mathrm{M} \mathrm{MgCl}_{2}$ & no dialysis & $10000^{a}$ & $+14^{a}$ & 110 \\
& 3 days & $590^{b}$ & -25 & 0.07 \\
& 6 days & $610^{b}$ & -29 & 0.03 \\
$0.2 \mathrm{mM} \mathrm{FeCl}_{3}$ & no dialysis & $10000^{a}$ & $+4^{a}$ & 2.5 \\
& 3 days & $570^{b}$ & -27 & 0.04 \\
& 6 days & $750^{b}$ & -27 & 0.04
\end{tabular}

${ }^{a_{T}}$ These are indicative values as size and measurement polydispersity are too large for accurate analysis. Sizes larger than roughly $1000 \mathrm{~nm}$ usually indicate macroscopic aggregation in this system. ${ }^{b}$ Stable systems.

than one year) stability has been emphasized. In general, all unstable samples had initial zeta potentials close to $-40 \mathrm{mV}$ that fell below $-30 \mathrm{mV}$ after one year, while the stable samples started out above $-50 \mathrm{mV}$ and ended up above $-40 \mathrm{mV}$. It is a well-known effect for electrocratic systems that the zeta potential can change sign upon the addition of background electrolyte due to specific adsorption, although this is usually found for multivalent ions. ${ }^{14,15}$

\section{DISCUSSION}

This section is divided in two parts discussing the two phenomena, as it is plausible that these are governed by separate, but related, mechanisms. These phenomena are (1) the repeptization of aggregated iron pyrophosphate and (2) the long-term stability of the dialyzed high salt system.

Repeptization. Colloidal iron pyrophosphate shows the characteristic properties for electrocratic systems like the dependence of zeta potential on background ionic strength ${ }^{13}$ and excess precursor salt (see Supporting Information). However, the repeptization reported here shows some important differences with that of well-studied redispersible electrocratic systems such as the silver iodide sols. ${ }^{3,16}$ First of all, the iron pyrophosphate system can be repeptized even after having aged for more than a month, while for most systems, the repeptization drastically decreases over time., ${ }^{2,3}$ Second, colloidal iron pyrophosphate can be repeptized when coagulated with multivalent ions, something that is impossible for other electrocratic systems. ${ }^{8,9,16}$ This unexpected repeptization of iron pyrophosphate is likely caused by the amorphous nature and open structure of the precipitate, which is observed independent of the preparation method. ${ }^{17}$ During coprecipitation of $\mathrm{FePP}_{\mathrm{i}}$, the 5-20 nm particles immediately form $\sim 200$ $\mathrm{nm}$ clusters that slowly aggregate further until they are macroscopically large. We have proposed that this slow further growth occurs by connecting the smaller, $5 \mathrm{~nm}$ particles that have a lower stabilizing barrier. ${ }^{13}$ In none of our (cryo-)TEM analyses have we found that the resulting open structure of the clusters collapses completely after full aggregation and aging of the system. The smallest particles will dissolve first during dialysis (also indicated in Figure $4 \mathrm{~b}, \mathrm{c}$ ) due to their higher solubility. ${ }^{15}$ Also, the interactions are very short ranged as we have shown before $(>10 \mathrm{~nm}){ }^{13}$ Therefore, removing the linking $5 \mathrm{~nm}$ particles will be sufficient to release the clusters from their aggregated state. This would avoid the need for a permanent cutoff distance that is often achieved by the addition 
Table 2. Dynamic Light Scattering, Zeta Potential, and Conductivity Results of the Low Salt and High Salt Systems

\begin{tabular}{|c|c|c|c|c|c|c|c|}
\hline \multirow[b]{2}{*}{ sample } & \multirow[b]{2}{*}{ dialysis duration } & \multicolumn{2}{|c|}{ size $(n m)$} & \multicolumn{2}{|c|}{ zeta potential $(\mathrm{mV})$} & \multicolumn{2}{|c|}{ conductivity $(\mathrm{mS} / \mathrm{cm})$} \\
\hline & & 0 days & 360 days & 0 days & 360 days & 0 days & 360 days \\
\hline \multirow[t]{3}{*}{ low salt } & no dialysis & $210^{b}$ & $4000^{a}$ & -37 & $-25^{a}$ & 2.20 & 2.4 \\
\hline & 3 days & $220^{b}$ & $1000^{a}$ & -35 & $-26^{a}$ & 0.02 & 0.146 \\
\hline & 6 days & $210^{b}$ & $1000^{a}$ & -38 & $-24^{a}$ & 0.02 & 0.085 \\
\hline \multirow[t]{3}{*}{$2 \mathrm{M} \mathrm{NaCl}$} & no dialysis & $4000^{a}$ & $14000^{a}$ & & $+8^{a}$ & & 163 \\
\hline & 3 days $^{b, c}$ & $440^{b}$ & $410^{b}$ & -55 & -43 & 0.03 & 0.111 \\
\hline & 6 days & $360^{b}$ & $2000^{a}$ & -37 & $-17^{a}$ & 0.01 & 0.080 \\
\hline
\end{tabular}

${ }^{a}$ Indicative values, size, and measurement polydispersity are too large for accurate analysis. Sizes larger than roughly $1000 \mathrm{~nm}$ usually indicate macroscopic aggregation in this system. ${ }^{b}$ Stable systems. ${ }^{c}$ The only preparation route that leads to systems still stable after one year $\left(\right.$ high salt $\mathrm{Na}^{+}$ dialyzed for 3 days). Note that this behavior has been observed in multiple samples, see Figure $4 a$.

of surfactants. The process is schematically summarized in Figure 5 .

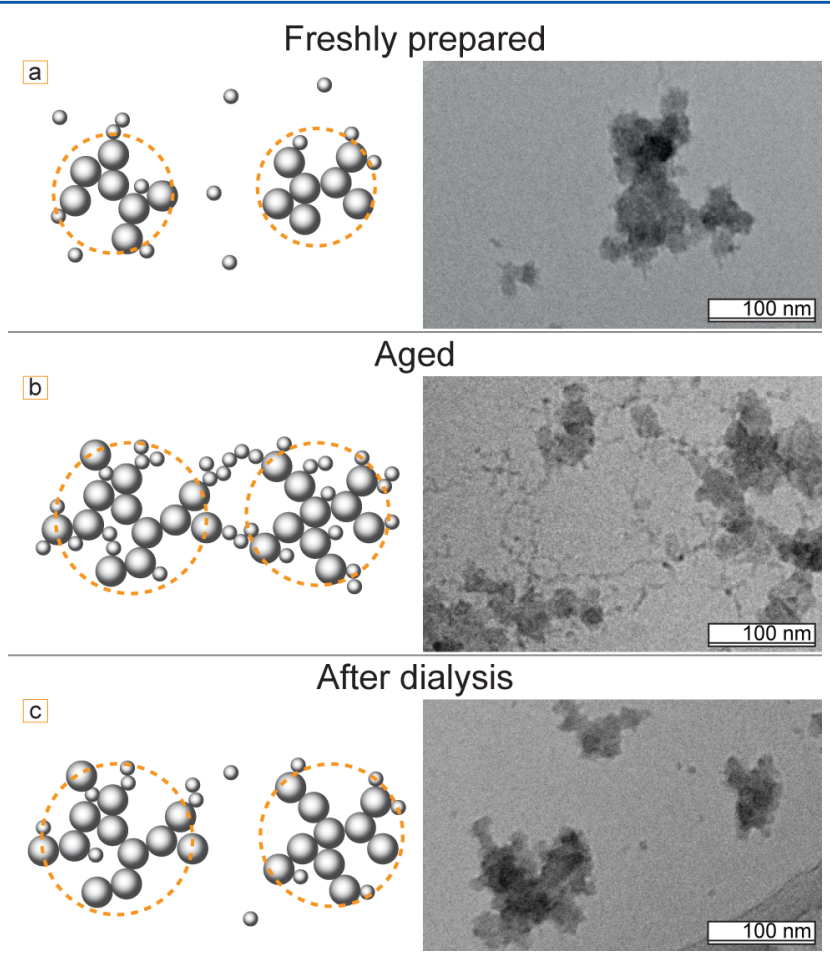

Figure 5. Repeptization by dissolution. The clusters formed after precipitation (a) are connected by the $5 \mathrm{~nm}$ nanoparticles during aging (b). During dialysis, the smallest particles will dissolve first, releasing the clusters from their aggregated state (c). Images on the right are from cryo-TEM analysis. TEM in panel $b$ reprinted with permission from ref 13. Copyright 2012 Elsevier.

Long-Term Stability. The results are schematically recapitulated in Figure 6, emphasizing the specific preparation route that leads to the system with long-term stability (d, highlighted in gray). The most obvious difference between the stable and unstable systems is the zeta potential: while the potentials and conductivities of Figure $6 c$,e,f are equal within error limits, the potentials of $\mathrm{c}$ and $\mathrm{d}$ are very far off while they are at similar conductivity. It is not immediately clear why a system prepared in this way yields a stronger zeta potential (d) or why it subsequently decreases again with longer dialysis time (f).

While we currently have no ready explanation for this phenomenon, the long-term stability of the high salt system dialyzed for three days is likely due to its stronger zeta-

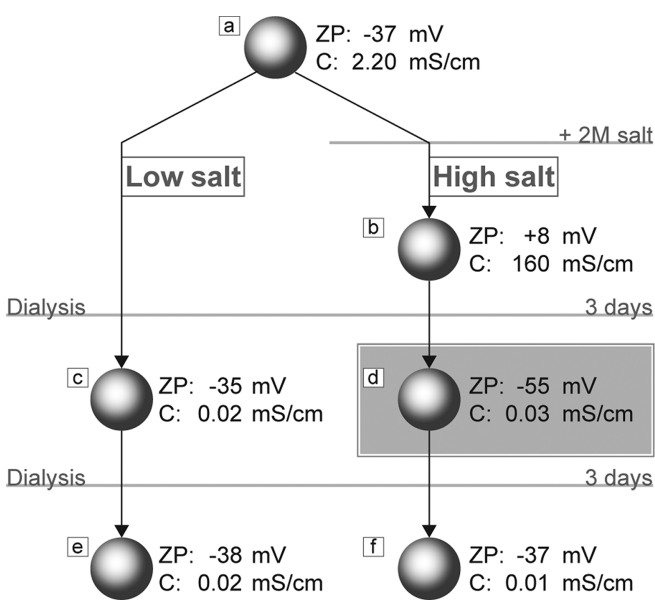

Figure 6. Schematic overview of the results. The freshly prepared system (a) is divided in two parts: one is left as-is; the other is adjusted to $2 \mathrm{M}$ salt (b). Both samples are dialyzed for three days $(\mathrm{c}, \mathrm{d})$ or six days (e,f). The only system with long-term stability ( $1+$ year) is highlighted in gray (d). Zeta potentials (ZP) and conductivities (C) are stated next to each sample.

potential: it is the only system with a zeta potential (ZP) below $-50 \mathrm{mV}$. We expect that this is caused by a combination of surface effects and the slight solubility of the material (see Supporting Information), and we consider the following model. In this model, some modification of the particle surface occurs at the highest ionic strength (Figure 6b). Possible causes for this are specific adsorption of background electrolyte, preferential dissolution of one of the components, or generation of extra surface charges facilitated by the strong screening at high ionic strength. However, in none of our previous studies have we found evidence for either specific adsorption of $\mathrm{Cl}^{-}$ions or preferential dissolution of $\mathrm{Fe}^{3+}$.

Upon dialysis (of three days), the excess electrolyte is removed, but the surface remains altered with the additional charge remaining on the particle, resulting in a more negative zeta-potential (comparing $\mathrm{c}$ and $\mathrm{d}$ ) and the observed long-term stability. Dialysis of another three days then etches the surface of the particles due to the slight solubility of the material, resulting in a clean surface similar to the low salt system (comparing e and $\mathrm{f}$ ).

To identify the mechanism behind this phenomenon, it would be insightful to know the exact surface composition of the particles at the various states in Figure 6. Unfortunately, because of the small size of the particles and the beamsensitivity of the material, ${ }^{17}$ we have yet to find a suitable technique to analyze this. 


\section{CONCLUSIONS}

We report two findings. (1) Colloidal systems of iron pyrophosphate destabilized by the addition of a large excess of salt can be repeptized by means of dialysis, independent of waiting time or concentration and valence of excess salt. This is surprising as the system shows most of the characteristics expected for an electrocratic system, and we have not seen this elsewhere in literature. We argue that this unexpected repeptization can be explained by the open structure and limited solubility of the material. (2) The stability of colloidal iron pyrophosphate can be increased by the addition of salt and subsequent dialysis of the sample, but excessive dialysis of such a sample again leads to stability comparable to a system dialyzed just after preparation. This is most likely due to the complex interplay between the experimental conditions and the zeta potential of slightly soluble materials, causing a stability optimum at a certain ionic strength and surface composition. Understanding of these two mechanisms will be helped greatly by analysis of the surface composition of the particles at various states during dissolution (for the repeptization) and at various compositions of the medium (for the long-term stability). However, because of the beam-sensitivity and small size of the particles, we have yet to find a suitable analysis technique for this.

\section{ASSOCIATED CONTENT}

\section{(S Supporting Information}

(i) High salt systems with $\mathrm{M}^{\mathrm{III}}$ as background ions. (ii) $\mathrm{Fe}$ or $\mathrm{PP}_{\mathrm{i}}$ deficient systems. (iii) Solubility of iron(III) pyrophosphate. This material is available free of charge via the Internet at http://pubs.acs.org.

\section{AUTHOR INFORMATION}

\section{Corresponding Author}

*Fax: +31-302533870. Phone: +31-302532873. E-mail: Y.M. vanleeuwen@uu.nl (Y.M.v.L.); W.K.Kegel@uu.nl (W.K.K.).

\section{Notes}

The authors declare no competing financial interest.

\section{ACKNOWLEDGMENTS}

We thank Agentschap NL for financial support; this work is financially supported by Food Nutrition Delta program grant FND07002.

\section{REFERENCES}

(1) Israelachvili, J. N. Intermolecular \& Surface Forces; Academic Press, Inc.: New York, 1985.

(2) Frens, G.; Overbeek, J. T. G. Repeptization and the Theory of Electrocratic Colloids. J. Colloid Interface Sci. 1972, 38, 376-387.

(3) Overbeek, J. T. G. Recent Developments in the Understanding of Colloid Stability. J. Colloid Interface Sci. 1977, 58, 408-422.

(4) Desset, S.; Spalla, O.; Cabane, B. Redispersion of Alumina Particles in Water. Langmuir 2000, 16, 10495-10508.

(5) Molina-Bolivar, J.; Galisteo-Gonzalez, F.; Hidalgo-Alvarez, R. Colloidal Aggregation in Energy Minima of Restricted Depth. J. Chem. Phys. 1999, 110, 5412-5420.

(6) Rohrsetzer, S.; Bán, S.; Kovács, P.; Pászli, I. Colloidal Stability of Electrostatically Stabilized Sol Particles. Part II: The Role of Hydration and the Concentration of Potential-Determining Ions in Coagulation and Peptization of Ferric Hydroxide Sols. Colloid Polym. Sci. 1995, 273, 189-194.

(7) Qi, L.; Sehgal, A.; Castaing, J. C.; Chapel, J. P.; Fresnais, J.; Berret, J. F.; Cousin, F. Redispersible Hybrid Nanopowders: Cerium
Oxide Nanoparticle Complexes with Phosphonated-PEG Oligomers. ACS Nano 2008, 2, 879-888.

(8) Poovarodom, S.; Berg, J. C. Spontaneous Repeptization of Colloidal Dispersions. Colloids Surf., A 2009, 342, 16-23.

(9) Wu, W.; Giese, R.; van Oss, C. Linkage between Sigma-Potential and Electron Donicity of Charged Polar Surfaces 0.2. Repeptization of Flocculation Caused by Plurivalent Counterions by Means of Complexing Agents. Colloids Surf., A 1994, 89, 253-262.

(10) Wegmüller, R.; Zimmermann, M. B.; Moretti, D.; Arnold, M.; Langhans, W.; Hurrell, R. F. Particle Size Reduction and Encapsulation Affect the Bioavailability of Ferric Pyrophosphate in Rats. J. Nutr. 2004, 134, 3301-3304.

(11) Fidler, M. C.; Walczyk, T.; Davidsson, L.; Zeder, C.; Sakaguchi, N.; Juneja, L. R.; Hurrell, R. F. A Micronised, Dispersible Ferric Pyrophosphate with High Relative Bioavailability in Man. Br. J. Nutr. 2004, 91, 107-112.

(12) Hilty, F. M.; Arnold, M.; Hilbe, M.; Teleki, A.; Knijnenburg, J. T. N.; Ehrensperger, F.; Hurrell, R. F.; Pratsinis, S. E.; Langhans, W.; Zimmermann, M. B. Iron from Nanocompounds Containing Iron and Zinc is Highly Bioavailable in Rats without Tissue Accumulation. Nat. Nanotechnol. 2010, 5, 374-380.

(13) van Leeuwen, Y. M.; Velikov, K. P.; Kegel, W. K. Stabilization through Precipitation in a System of Colloidal Iron(III) Pyrophosphate Salts. J. Colloid Interface Sci. 2012, 38, 43-47.

(14) Schneider, C.; Hanisch, M.; Wedel, B.; Jusufi, A.; Ballauff, M. Experimental Study of Electrostatically Stabilized Colloidal Particles: Colloidal Stability and Charge Reversal. J. Colloid Interface Sci. 2011, $358,62-67$

(15) Hunter, R. J. Foundations of Colloid Science; Oxford University Press: Oxford, U.K., 2001.

(16) Frens, G.; Overbeek, J. T. G. Repeptization of AgI-Flocs. J. Colloid Interface Sci. 1971, 36, 286-288.

(17) van Leeuwen, Y. M.; Velikov, K. P.; Kegel, W. K. Morphology of Colloidal Metal Pyrophosphate Salts. RSC Adv. 2012, 2, 2534-2540. 